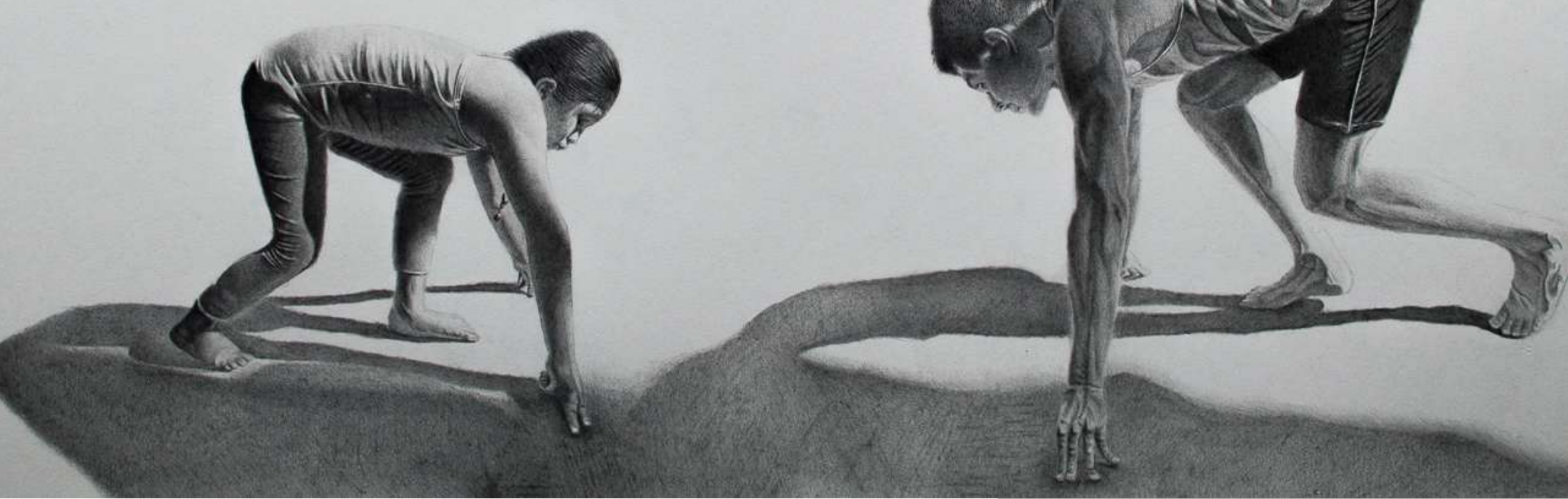

Sin título

De la serie Un viaje hacía el origen

Grafito sobre papel

$15 \times 50 \mathrm{~cm}$

2019

Medellín 


\title{
Modelando las políticas públicas. Estrategias de las organizaciones de base para la incidencia en políticas públicas. Un estudio de casos del Área Metropolitana de Buenos Aires, Argentina*
}

\author{
Itatí del Rosario Moreno (Argentina)**
}

\section{Resumen}

Existen múltiples actores que inciden en las políticas públicas, entre estos se encuentran las organizaciones de base. Muchas de estas organizaciones, a pesar de no contar con grandes recursos, logran con éxito su objetivo e inciden en las políticas públicas, dejando su marca en la configuración de prioridades y políticas estatales. Ante esto, es pertinente preguntarse no solo a qué estrategias de acción recurren las organizaciones de base para incidir en las políticas públicas, sino también de qué manera estas estrategias pueden favorecer dicha incidencia. A partir del estudio de casos y la comparación como principales métodos de trabajo, el argumento de este artículo es que la combinación sostenida en el tiempo de estrategias de mediación, de confrontación y de expansión de la arena de decisión favorece a la incidencia en las políticas públicas de las organizaciones de base, ya que permiten diferentes formas de acceso a las esferas de decisión.

\section{Palabras clave}

Políticas Públicas; Organizaciones de Base; Política Local; Estrategias Políticas; Agregación de Intereses; Incidencia Política.

Fecha de recepción: febrero de 2019

- $\quad$ Fecha de aprobación: julio de 2019

\section{Cómo citar este artículo}

Moreno, Itatí del Rosario. (2019). Modelando las políticas públicas. Estrategias de las organizaciones de base para la incidencia en políticas públicas. Un estudio de casos del Área Metropolitana de Buenos Aires, Argentina. Estudios Políticos (Universidad de Antioquia), 56, pp. 67-90. DOI: 10.17533/udea.espo.n56a04

\footnotetext{
* Este artículo se deriva de la investigación Cambiando el rumbo: organizaciones de base contra rellenos sanitarios y sus efectos en las políticas de residuos de la Región Metropolitana de Buenos Aires, 2014-2019, en el marco del Doctorado en Ciencia Política, Escuela de Política y Gobierno, Universidad Nacional de San Martín, Argentina.

** Licenciada en Ciencia Política. Magíster en Derechos Humanos y Democratización para América Latina. Docente investigadora de la Escuela de Política y Gobierno y miembro del Grupo Ambiente y Política, Universidad Nacional de San Martín, Argentina. Correo electrónico: itati.moreno@yahoo. com.ar - Orcid: 0000-0002-9489-4775
} 


\title{
Shaping Public Policies. Grassroots Organizations' Strategies to Incidence on Public Policy. A Cases Study in Metropolitan Area of Buenos Aires, Argentina
}

\begin{abstract}
Several actors tend to influence public policies. Among these groups, we can find grassroots organizations. Many of these organizations, despite not having great resources, successfully achieve their political aims and influence public policies, leaving their mark on the configuration of State priorities and policies. In this context, it is relevant to ask not only what action strategies do grassroots organizations use to influence public policies, but also how strategies favor this incidence. The results of the case studies and the implementation of the comparative method show that the combined use of mediation and, confrontation strategies, as well as the expansion of deliberative spaces, over time, further the incidence of grassroots organizations into the making of public policies, since they allow different ways of accessing to the decision making sphere.
\end{abstract}

\section{Keywords}

Public Politics; Grassroots Organizations; Local Politics; Political Strategies; Interest Aggregation; Political Incidence. 
Modelando las políticas públicas. Estrategias de las organizaciones de base...

\section{Introducción}

Las organizaciones de base son organizaciones que actúan en el ámbito local o en torno a problemas locales y están compuestas, principalmente, por vecinos, trabajadores, desocupados o emprendedores de diferente tipo (Uphoff, 1993; Bryant y Bailey, 1997). Algunas de estas organizaciones no solo demandan cambios en torno a diferentes áreas de la política pública, sino que muchas logran incidir en estas generando efectos estatales concretos a partir de su accionar. Con énfasis en este último punto y teniendo en cuenta que las organizaciones de base son actores que a priori no suelen contar con grandes recursos o redes para llevar adelante sus objetivos, resulta relevante observar qué hacen estas organizaciones para lograr incidir en las políticas, indagando sobre las estrategias de acción que despliegan las organizaciones de base para incidir en las políticas públicas y de qué manera estas estrategias pueden favorecer dicha incidencia.

Con este propósito se estudian dos experiencias de incidencia en torno a la política de residuos de una metrópolis latinoamericana cuya población y generación de residuos está aumentando notablemente en los últimos años: la Región Metropolitana de Buenos Aires (RMBA). Puntualmente, se analizan dos casos de organizaciones de base, un tipo de organización especialmente importante en los reclamos ambientalistas en América Latina (Cortez y Maillet, 2018; Merlinsky, 2013; Moore, 2009). Las organizaciones aquí estudiadas reclaman contra los rellenos sanitarios ubicados en sus lugares de origen, es decir, contra sitios de disposición final de residuos domiciliarios, exigiendo que se clausuren de forma permanente. En ambos casos se señalan impactos ambientales negativos como el principal sustento del reclamo. En uno de estos casos, como resultado de las acciones de incidencia, se modificó la política de residuos implementada en el territorio, provocando el cierre del relleno sanitario cuestionado y la inauguración, en su lugar, de una planta de tratamiento de residuos. En el otro caso, en cambio, no hubo grandes modificaciones en la política en cuestión, el cierre del relleno sanitario no se concretó y solo se estableció una mayor restricción respecto de los distritos que podían disponer sus residuos en ese predio.

Lo llamativo de estas organizaciones es que logran distintos tipos de cambios en la implementación territorial de una política pública que era común para todos los distritos de la región metropolitana estudiada. En este sentido, Ilaman también la atención los puntos en común entre estas 
organizaciones de base: a) reclaman contra la misma política y los mismos organismos regionales; b) poseen un objetivo común, el cierre de un relleno sanitario; y c) actúan en una escala geográfica y temporalidad análoga con recursos materiales semejantes.

La existencia de estos puntos comunes entre las organizaciones de cada caso y sus reclamos son indicativos de notorias semejanzas en términos estructurales y contextuales en ambas experiencias, por lo cual se torna de interés el análisis de aquello que hacen o dejan de hacer estos actores para poder incidir en las políticas públicas y cambiar su rumbo. En línea con ello, se observa que las estrategias desplegadas por las organizaciones analizadas emergieron como un aspecto diferenciador de los procesos de incidencia. El argumento principal de este artículo plantea que hay tres clases de estrategias de acción utilizadas por las organizaciones de base para incidir en las políticas públicas —estrategias de mediación, de confrontación y de expansión de la arena de decisión- y que sostener en el tiempo un uso combinado de estas diferentes estrategias favorece una mayor incidencia en las políticas públicas. Si bien este argumento encierra una inferencia válida para los casos estudiados, es factible de replicarse en estudios sobre otras organizaciones de base que pretenden incidir en otros tipos de políticas en

[ 70 ] condiciones semejantes.

En lo que refiere a la recolección de los datos, se utilizaron técnicas cualitativas como la entrevista en profundidad a más de una veintena de personas - entre activistas y funcionarios-, la observación participante de reuniones de las organizaciones estudiadas y el análisis de documentos generados por ellas, por la prensa y por organismos estatales. El periodo analizado es del 2003 hasta la actualidad.

\section{Incidencia política: la tarea de forjar cambios en las políticas públicas}

Los trabajos sobre incidencia política, de manera más o menos explícita, abordan la cuestión del cambio en las políticas públicas. Estos trabajos están especialmente focalizados en describir o explicar cómo los actores pueden provocar modificaciones en el rumbo de la gestión de las políticas públicas (Burstein y Linton, 2002; Cortez y Maillet, 2018; Ydersbond, 2014). Por ello, y con base en las definiciones propuestas por José Ascención Moreno y Rosa Amelia Arballo, (2003) y Paul Burstein, Rachel Einwohner y Jocelyn 
Hollander (1995), se considera apropiado definir a la incidencia en políticas públicas como aquellos efectos estatales - buscados y no buscadosgenerados por actores que activamente intentan influenciar el rumbo de las políticas públicas de un área de gestión específica.

Por efectos estatales se refiere al surgimiento de decisiones públicas orientadas al desarrollo de nuevos programas de política pública o modificaciones en los programas vigentes. Estas modificaciones se pueden observar en varias dimensiones de esa política, entre las que se encuentran los momentos lógicos de esta —el diseño, la implementación, el monitoreo o la evaluación-, los instrumentos de política pública utilizados —instrumentos de regulación, económicos y de información (Vedung, 1998)—, los objetivos y los valores básicos que la orientan, o los actores u organismos encargados de implementarla o monitorearla (Capano y Howlett, 2009).

En caso de que el proceso de incidencia arroje resultados, se pueden observar distintos tipos de cambio en las políticas públicas. Estos cambios pueden ubicarse en un continuo que va desde una menor a una mayor incidencia y que incluye desde cambios en la política vigente hasta un cambio de política, es decir, la generación de una nueva política pública.

Los procesos de incidencia no se explican por la sola movilización y demanda de unos actores y su presión sobre los decisores públicos. Estos intentos y preferencias de cambio en la gestión pública no se traducen de forma lineal en modificaciones en el ámbito público. Estas instancias de continuidad de las políticas vigentes no resultan extrañas para aquellos estudios que enfatizan que las políticas públicas, por lo general, están más signadas por la permanencia que por grandes transformaciones (Lindblom, 1992; Pierson y Skocpol, 2008). Sin embargo, existe una vasta literatura sobre políticas públicas, policy change y movimientos y organizaciones sociales que señalan lo contrario y da un lugar preponderante al análisis de las transformaciones de las políticas públicas y, sobre todo, a aquellas derivadas de procesos de incidencia de distintos tipos de actores. Entre estos trabajos se encuentran, por ejemplo, aquellos que aluden a cambios en políticas públicas generados por la influencia de grupos de protesta o movimientos sociales (Andretta, Piazza, y Subirats, 2015; Burstein, Einwohner y Hollander, 1995; Steedly y Foley, 1979), coaliciones promotoras de ideas (Jenkins-Smith y Sabatier, 1994; Weible et al., 2011), grupos empresariales o sindicatos (Offe y Wiesenthal, 1980), grupos de lobby (Leech, 2010; Whiteley y Winyard, 
1987) u organizaciones de base -como las aquí estudiadas- (Bullard y Johnson, 2000; Walsh, Warland y Smith, 1997), entre otros actores.

Estos grupos pueden encontrarse especialmente limitados en sus objetivos de incidencia política por cuestiones estructurales como, por ejemplo, la situación macroeconómica nacional, la estructura productiva o la estructura de clases (Offe y Wiesenthal, 1980), la estructura de oportunidades políticas (Tarrow, 2011), la aparición de «ventanas de oportunidad» (Kingdon, 1995) y coyunturas críticas (Mahoney, 2001; Pierson, 1993), o las capacidades estatales que existan para responder el reclamo en el área de gestión a la que estén interpelando (Evans, Rueschemeyer y Skocpol, 1985; Mann, 2008). Sin embargo, muchas veces los condicionantes estructurales no operan de forma decisiva en algunos procesos de incidencia y terminan adquiriendo mayor peso explicativo otros factores causales como características de los agentes movilizados o de las acciones que llevan adelante.

Entre estas explicaciones se pueden encontrar aquellas que atribuyen la generación de resultados estatales a aquello que poseen los actores movilizados o a aquello que hacen. En el primer caso, se pueden encontrar los trabajos que enfatizan la relevancia de los recursos materiales,

[ 72 ] organizacionales y humanos (Burstein y Linton, 2002; McCarthy y Wolfson, 1996; Ward, 2017), o - especialmente relevante en conflictos ambientales, por ejemplo- el recurso de la información (Sabatini, 1997) con que cuentan los actores. En el segundo caso, se pueden señalar los trabajos sobre las estrategias de acción que despliegan los actores, los cuales argumentan que la incidencia está condicionada por la manera en que los actores se relacionan con los decisores públicos (Molina, 2017, 26-28 de julio; König, Tsebelis y Debus, 2011).

Algunos autores enfatizan que las estrategias que más favorecen la incidencia de la acción colectiva son las que hacen uso con alta frecuencia de métodos disruptivos, como la protesta o la movilización (McAdam y Su, 2002), lo cual, en ocasiones, puede incluir el uso de violencia (Young, 2016), aunque sobre este punto no hay acuerdo en la literatura (McAdam y $\mathrm{Su}, 2002)$. Otros trabajos, en cambio, señalan las virtudes de la utilización de los canales institucionales (Christel y Gutiérrez, 2017; Gutiérrez e Isuani, 2014) y la cooperación con los decisores (Mitlin, 2008; Najam, 2000; Sorroche, 2017). 
Este último grupo de explicaciones de la incidencia política, las centradas en las estrategias de acción, son especialmente adecuadas para el abordaje de los dos casos de estudio analizados en este trabajo. En ambos casos son organizaciones de base semejantes las que emprenden el proceso de incidencia para intentar torcer el rumbo de una política pública que se implementa en el ámbito regional en el área metropolitana que ambos grupos habitan. Esto conlleva, al menos, dos implicancias, debido a que, por un lado, los actores de cada caso cuentan con recursos semejantes y, por otro lado, operan en un contexto regional común bajo condiciones estructurales políticas, sociales y culturales también análogas. En consecuencia, las hipótesis explicativas que apuesten por factores causales vinculados a aspectos como los recursos o las condiciones estructurales parecen ser menos apropiadas para este estudio que las que resalten la relevancia de las estrategias de acción desplegadas por los actores movilizados. En línea con ello, este artículo se centra en describir y clasificar las estrategias de acción llevadas adelante por las organizaciones de base estudiadas ya que, además de lo señalado, se destacan como aspectos distintivos en ambos casos. Conjuntamente, a partir de la comparación de ambas experiencias, se analizan cuáles son más propicias para llevar adelante procesos de incidencia con resultados favorables.

\section{Estrategias para la incidencia en las políticas públicas: hacia una tipología}

Los actores que buscan incidir en las políticas públicas necesariamente deben interactuar con los decisores de las áreas de política de su interés, ya sea que se trate de funcionarios políticos, burócratas o técnicos. Esta interacción, según la literatura, puede darse a través de diversos tipos de estrategias que los actores despliegan con el fin de presionar a los decisores. Entre las distintas estrategias para la incidencia que estos actores -en particular, las organizaciones de base-despliegan, se identifican: a) estrategias de mediación, b) estrategias de confrontación y c) estrategias de expansión de la arena de decisión.

Las estrategias de mediación buscan convencer a un decisor para que apoye y adopte determinada propuesta política a través de acciones no confrontativas y principalmente dialoguistas con el fin de vincular demanda y política (Diani, 2003; Tilly, 2001). Así, los actores recurren a la entrega de petitorios, el pedido de audiencias o reuniones, el seguimiento activo de 
los reclamos y estrategias afines para las que se utilizan principalmente los canales institucionales de comunicación (Gutiérrez e Isuani, 2014).

Las estrategias de confrontación están orientadas, como su nombre lo indica, al enfrentamiento directo con el decisor para que revierta la política pública de interés para el actor movilizado. Este tipo de estrategia puede manifestarse en forma de protestas, movilizaciones u otros actos de irrupción pública semejantes realizados en contra del decisor en cuestión (Tarrow, 2011; Tarrow y Tilly, 2009), así como en estrategias de presión a través de campañas públicas y mediáticas (Kotler y Zaltman, 1971).

En tercer lugar, se encuentran las estrategias de expansión de la arena de decisión, que son aquellas en donde los actores tratan de ampliar el locus político e institucional donde se define la política pública, ya sea de forma horizontal -involucrando otras áreas del mismo ámbito gubernamentalo de forma vertical -involucrando áreas de ámbitos gubernamentales superiores- (Boucher, 2013). Los actores se mueven entre diferentes arenas o instancias institucionales con el fin de lograr sus objetivos de política pública en una instancia estatal más favorable para sus propuestas (Pralle, 2003; Real-Dato, 2009). Esos entornos institucionales más favorables

[ 74 ] pueden encontrarse dentro de otras áreas u organismos del Poder Ejecutivo o fuera de él, como, por ejemplo, las legislaturas, donde se pueden lograr cambios en las políticas a través de la modificación de las normas existentes o la sanción de nuevas (Pralle, 2006). Asimismo, otra estrategia para la expansión de las áreas de decisión de política pública es la judicialización de la política (Delamata, 2016; Ryan, 2011), donde se busca activamente la intervención de cortes judiciales para presionar desde esa arena a los ejecutivos y concretar así nuevas formas de gestionar en política pública.

Entendiendo la naturaleza relacional y dinámica de las arenas de gestión estatal (Lowi, 1996; Real-Dato, 2009; True, Jones y Baumgartner, 2010), las estrategias de mediación y de expansión de la arena de decisión cobran especial importancia como mecanismos que permiten la apertura de canales de influencia sobre las áreas de decisión de interés, pudiendo llegar, incluso, a superar a la confrontación como única estrategia de acción. Asimismo, retomando la idea de que los cambios no se dan de forma espontánea, se suele tornar necesario para los actores desarrollar la capacidad de sostener en el tiempo las estrategias de incidencia que despliegan. 
En línea con lo expuesto anteriormente y con lo analizado en los casos, sostener en el tiempo un uso combinado de estas diferentes estrategias de mediación, de confrontación y de expansión de la arena de decisiónen procesos de incidencia de organizaciones de base favorece la mayor incidencia en las políticas públicas.

Las organizaciones de base en acción contra la política de residuos de la Región Metropolitana de Buenos Aires

Los residuos domiciliarios de la RMBA son recolectados por los municipios y trasladados hasta rellenos sanitarios gestionados por la empresa pública Coordinación Ecológica Área Metropolitana Sociedad del Estado (Ceamse), donde son finalmente enterrados. En 2003 existían cuatro rellenos sanitarios en funcionamiento - Villa Domínico, González Catán, de Punta Lara y de Norte III- para recibir los residuos domiciliarios generados por toda la RMBA: 4254 778,60 toneladas por año, es decir, 11 818,83 toneladas diarias (véase mapa 1).

Mapa 1. Región Metropolitana de Buenos Aires (RMBA) con división política municipal y rellenos sanitarios activos en 2003.

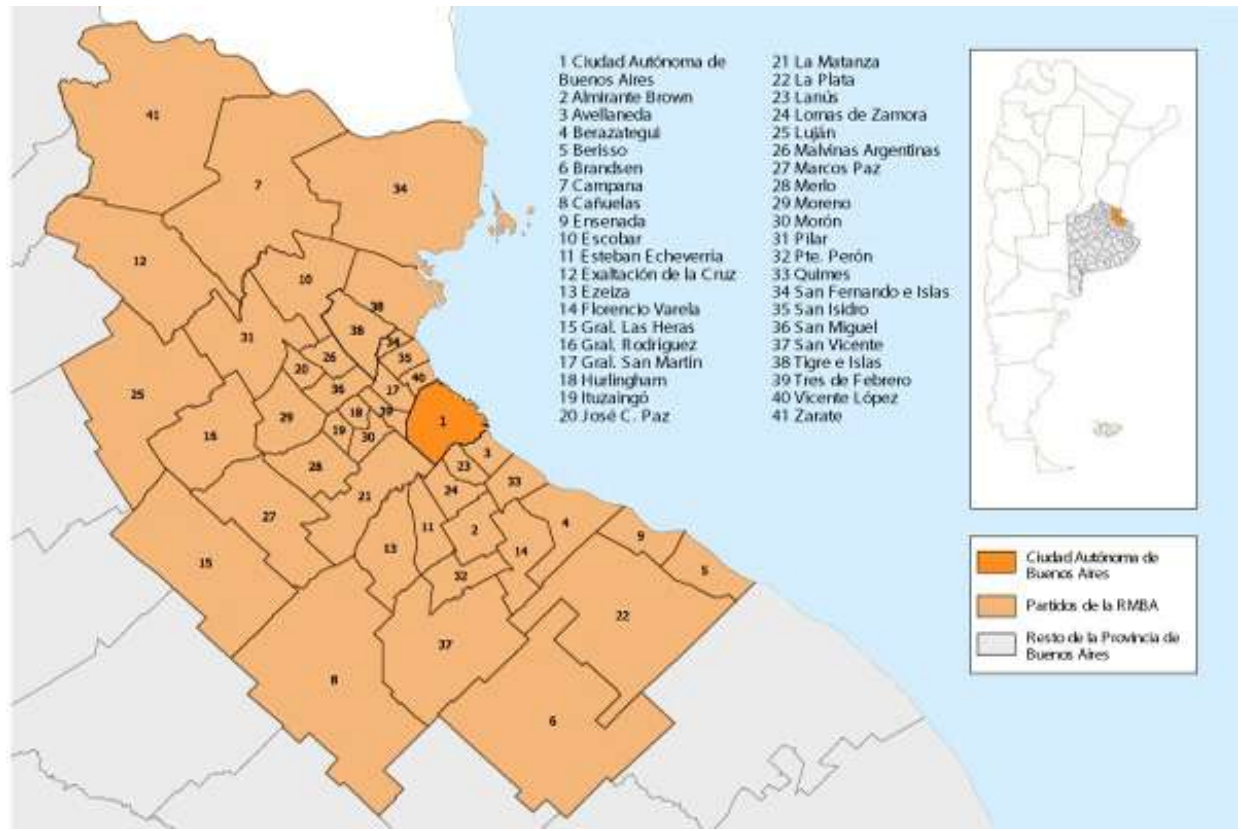

Fuente: tomado de Observatorio del Conurbano Bonaerense (s. f). 
En torno a estos cuatro rellenos sanitarios se registraron protestas y reclamos de cierre por parte de organizaciones de base locales de cada territorio. Los primeros reclamos en contra de un relleno se desarrollaron en 2003-2004 (Villa Domínico) y los últimos se dieron principalmente en 2012-2013 (Norte III). En esta ocasión se pretende avanzar hacia una caracterización y comparación de los reclamos de González Catán y Punta Lara, los cuales son más comparables entre sí porque efectúan un mismo reclamo - cierre del relleno y creación de políticas de gestión integral de residuos-, ambos se desarrollan en una temporalidad común —desde 2003 hasta la actualidad- y en una misma escala regional - Región Metropolitana de Buenos Aires-; ambos reclamos son Ilevados adelante por organizaciones de base y se desencadenan ante la percepción de mayor riesgo ambiental asociado a la presencia del relleno sanitario en cada ciudad; en ambos casos, el principal actor estatal interpelado fue la empresa gestora del relleno, Coordinación Ecológica Área Metropolitana Sociedad del Estado, y adicionalmente, contra los gobiernos provincial y municipal —del mismo partido y signo político en ambos casos-. ${ }^{1}$

Algo que diferencia a estas organizaciones son las estrategias que despliegan para incidir en la política de residuos. La organización de González Catán -Asamblea de Vecinos Autoconvocados contra la Ceamsepriorizó el uso de estrategias de confrontación y solo eventualmente utilizó una estrategia de extensión de la arena de decisión o de mediación sin uso sostenido de ellas. La organización de base contra el relleno de Punta Lara -Nuevo Ambiente- apostó al uso combinado y sostenido en el tiempo de estrategias de mediación, confrontación y extensión de la arena de decisión. Ambas organizaciones incidieron de diferente manera en la política de residuos. En el caso de González de Catán, las autoridades de la Ceamse y las provinciales modificaron, en parte, la implementación de la política de residuos vigente, reduciendo el ingreso de residuos al relleno pero sin cerrarlo. En el caso de Punta Lara, las mismas autoridades decidieron el cierre del relleno y construyeron una planta de tratamiento de residuos que

\footnotetext{
${ }^{1}$ En la estructura federal argentina existen tres jurisdicciones gubernamentales: nacional, provincial y municipal. La municipal se encarga de la gestión de los residuos, aunque en algunos casos la disposición final de los residuos está en manos de organismos regionales — supramunicipales, específicamenteo provinciales. Este es el caso de la RMBA, donde los municipios están a cargo de la recolección de los residuos domiciliarios y una empresa de esa área metropolitana — la Ceamse - está encargada del enterramiento final de los residuos en sus rellenos sanitarios. Esta empresa pertenece en $50 \%$ al gobierno provincial y es controlada por organismos ambientales provinciales: el Organismo Provincial para el Desarrollo Sostenible (OPDS) y la Autoridad del Agua (ADA).
} 
opera desde 2017. Además, las autoridades locales implementaron una nueva política de residuos orientada a la recuperación de materiales.

Es Ilamativo que los mismos actores estatales que decidieron instaurar una nueva política de residuos — cerrar el predio de Punta Lara y construir una planta de tratamiento final- en otro caso no lo hayan hecho y solo hayan modificado parcialmente la implementación de la política —reducir la cantidad de residuos que llegan al relleno de González Catán-.

\subsection{Caso González Catán: Asamblea de Vecinos Autoconvocados contra la Ceamse de González Catán}

En 2003 algunos vecinos comenzaron a organizarse ante un incremento de enfermedades cancerígenas entre los habitantes de las inmediaciones del predio de la Ceamse, a la cual señalaban como responsable de contaminar el ambiente y afectar la salud de la población. De esa incipiente organización vecinal nació en el mismo año la Asamblea de Vecinos Autoconvocados contra la Ceamse. El principal reclamo de esta organización de base fue el cierre del relleno y el pedido de políticas de gestión integral de residuos para el distrito donde estaba el relleno - Municipio de La Matanza-, el distrito que mayor cantidad de residuos enviaba a ese predio.

En uno de los principales documentos desarrollados por la Asamblea, Programa Catán Sin Basura, expusieron claramente la propuesta programática de la organización, la cual exhortaba a las autoridades a la implementación de políticas para el reciclado de residuos orgánicos, plásticos, papeles, metales y cartón: «Entendemos que la propuesta del reciclado de la basura de González Catán parte necesariamente de una condición irrenunciable para el pueblo que es el cierre y saneamiento ya de la Ceamse de nuestra localidad» (Asamblea de Vecinos Autoconvocados, 2007, p. 3).

Las acciones que la Asamblea desarrolló desde el primer momento interpelaron directamente a tres actores: a la Ceamse, al gobierno provincial y al gobierno municipal, que según la organización de base «permitían» la presencia del relleno en el distrito. Desde la Asamblea no desplegaron estrategias de mediación, ya que el contacto con las autoridades ejecutivas solo se limitó a la presentación de algunas notas y reclamos institucionales. Para visibilizar sus demandas, el principal tipo de estrategia utilizada fue la confrontación, recurriendo típicamente a acciones contenciosas como la 
movilización, la protesta, el corte de calles, y los «bloqueos» en las puertas del relleno. Varios integrantes que participaron desde 2003 en la Asamblea manifestaron en las entrevistas realizadas la mayor confianza por las acciones contenciosas frente a las de otro tipo:

La forma de protesta es la única para que nos den bolilla. Aparte, [si] te metés con la política y la Justicia... no son independientes. La dejan ser independiente en lo que quieren. O sea, nosotros, si tiramos algo que molestaba al Gobierno, no le iban a dar bolilla (comunicación personal, Integrante de la Asamblea, González Catán, 27 de agosto, 2016).

Yo prefería más el bloqueo, a mí me parecía mejor, la marcha, el bloqueo (comunicación personal, Integrante de la Asamblea, González Catán, 30 de agosto, 2016).

En general, no hubo en esta Asamblea despliegue de estrategias de expansión de las arenas de decisión o mediación, salvo en dos ocasiones puntuales entre 2005 y 2007 en la justicia y en el legislativo local, las cuales eran siempre complementarias a la estrategia principal: la confrontación.

Entre 2006 y 2007 se dieron los principales actos de movilización y protesta de todo este proceso, a su vez, fue un momento en que la Asamblea activamente se dedicó a otro tipo de estrategia y de forma más sostenida, aunque por poco tiempo. En 2005 se inició un proceso de judicialización al denunciar penalmente a la Ceamse, acusándola de infringir la ley nacional de manejo de residuos peligrosos. Esta denuncia dio origen a la Causa Penal N. ${ }^{\circ}$ 299316 de junio de 2006 del Juzgado Federal en lo Criminal y Correccional N. ${ }^{\circ} 3$ de Morón. El uso de la estrategia de judicialización derivó en 2006 en el primer fallo judicial: el agua de la zona estaba contaminada, debía proveerse agua potable a las escuelas públicas de la zona, profundizarse la investigación al respecto y que la Ceamse y las autoridades municipales y provinciales debían presentar información respecto de las autorizaciones de vuelco de la empresa.

Más allá de que la contaminación en las cercanías al relleno sanitario y la existencia de conductos ilegales para el vuelco de los líquidos lixiviados quedaron demostradas, nunca se concretó una sentencia firme. Así lo expresaba una entrevistada que también destacaba que la judicialización no era seguida tan de cerca por los vecinos movilizados, es decir, que no era 
una estrategia por la que se apostaba de forma sostenida en el tiempo, como sí lo era la confrontación:

Si bien hay un fallo en la causa 2993, no hay una sentencia. Entonces en cualquier momento corremos el riego que la causa sea archivada sin sentencia [...]. El tema es que pasaron ya tres años y casi dos meses y la causa sigue abierta en el mismo estado: ni la archivaron, ni la sentenciaron, ni la paralizaron. Porque la causa está en letra, pero está ahí [...]. Pasa el tiempo y acumula polvo [...] a no ser por algún vecino que va de vez en cuando, tal vez no tan seguido como se debería ir, pero bueno, vamos, preguntamos, la respuesta es siempre la misma: «el juez está investigando». Pero nosotros, el hecho concreto es que el resultado de las investigaciones no lo estamos viendo (comunicación personal, Integrante de la Asamblea, González Catán, 18 de noviembre, 2013. Énfasis agregado).

Como se temía, ante ese seguimiento que «tal vez» no era tan frecuente "como debería ser», efectivamente la causa quedó archivada al cumplirse los diez años. El proceso de judicialización iniciado en 2005 tuvo algunas medidas favorables cuando el proceso era reciente y más «seguido» por los actores. En 2007, desde el fuero administrativo ordenaron el cierre del relleno sanitario, aunque el fallo se cumplió solo parcialmente: el ingreso de residuos de otros municipios quedó prohibido y el relleno quedó habilitado solo para los desechos provenientes del municipio de La Matanza, lo cual terminó siendo uno de los principales logros en términos de incidencia de la Asamblea que, para esos momentos, supo combinar el proceso judicial con el desarrollo de varias movilizaciones, protestas y bloqueos (ANRed, 2006, enero 12; Alarcón, 2006, julio 22; La Nación, 2006, noviembre 3; El Día, 2007, septiembre 12).

Conjuntamente, en este periodo de mayor movilización (20062007), la Asamblea apostó, aunque solo como una acción puntualizada, a la presentación de propuestas legislativas en el órgano legislativo local: en 2007 presentó un proyecto para implementar una política de gestión integral en el ámbito local, aunque la propuesta nunca fue tratada en el recinto y quedó solo como un expediente más (Expediente N. ${ }^{\circ} 1000$ de 2 de noviembre de 2007). La Asamblea no contaba con apoyos al interior del órgano legislativo ni gestó estrategias de mediación o alianzas con los miembros de este, los cuales, en su mayoría, no se mostraron interesados por la propuesta: 
Con el Expediente $\mathrm{N}^{\circ} 1000$ del 2 de noviembre del 2007 [...] una de las pocas cosas que hicieron estos concejales fue sacar una minuta de comunicación y mandarlo al Ejecutivo. Y el Ejecutivo ya lo tenía porque nosotros lo presentamos ahí también. Pero, en realidad, siempre lo archivaron, nunca les interesó. Acá para nosotros, los Autoconvocados, queda claro que la estructura del Estado municipal, nacional, provincial y de la Ciudad Autónoma protege el negocio de las empresas privadas (comunicación personal, Integrante de la Asamblea, González Catán, 3 de octubre 2013).

Como se puede observar, existe en la Asamblea cierto descrédito hacia las autoridades gubernamentales involucradas, la cual sostiene, en parte, la falta de apuestas por estrategias de mediación con estos actores.

Las demandas de la Asamblea tuvieron como única respuesta la restricción del ingreso de residuos al relleno sanitario de González Catán y no hubo avances hacia una gestión integral de los residuos o cierre del predio. Los decisores locales solo desarrollaron algunas experiencias puntuales y focalizadas de recolección diferenciada que fueron interrumpidas prontamente. Asimismo, el oficialismo local desarrolló y aprobó en 2010 una polémica normativa local que habilitaba la construcción de una planta de tratamiento de residuos con financiamiento de una empresa pública energética nacional, Energía Argentina Sociedad Anónima (Enarsa) (El1Digital, 2010, septiembre 20), la cual fue fuertemente rechazada por las organizaciones locales por proponer tecnologías experimentales asimilables a la incineración (comunicación personal, Integrante de la Asamblea, González Catán, 18 de noviembre 2013). El proyecto nunca se implementó, a pesar de que el municipio recibió gran parte del financiamiento, pero no lo destinó a la construcción de la proyectada planta (Semanario Quinto Poder, 2015, marzo 2). En la actualidad, la Asamblea sigue desarrollando protestas exigiendo el cierre del relleno sanitario y la implementación de nuevas políticas de residuos.

\subsection{Caso Punta Lara. Nuevo Ambiente}

Nuevo Ambiente es una pequeña organización no gubernamental compuesta por algunos expertos y vecinos ambientalistas dedicados a la protección de las especies vegetales y animales de la zona periurbana que habitan. ${ }^{2}$ En 2003, Nuevo Ambiente se involucró directamente en la

${ }^{2}$ Para mayor información, véase https://www.nuevoambiente.org/quienes-somos/ 
cuestión de los residuos cuando incrementó abruptamente la cantidad de desechos que ingresaban al relleno sanitario de Punta Lara, los cuales, con anterioridad, eran enterrados en el relleno de Villa Domínico (El Día, 2003, diciembre 6), clausurado de forma definitiva en 2004 (Aranda, 2004, enero 31). El ingreso de esos nuevos residuos provocó que las autoridades de la Ceamse ordenaran la ampliación del relleno.

Ante esto, Nuevo Ambiente comenzó a reclamar contra el ingreso de esos residuos y contra las obras de ampliación del relleno (El Día, 2003, diciembre 6). Para la organización, no se habían hecho las evaluaciones ambientales necesarias para habilitar el aumento del ingreso de desechos o la ampliación del predio señalando, además de que era ilegal la llegada de desechos provenientes de distritos ubicados a más de veinte kilómetros de distancia, de acuerdo al Decreto N. ${ }^{\circ}$ 9111/1978 de la provincia de Buenos Aires (Nuevo Ambiente, s. f.).

La organización exigió la realización de estudios de impacto ambiental y sus inmediaciones, la clausura de las ampliaciones del predio, la prohibición del ingreso de residuos de municipios alejados y el cierre total del relleno, para lo cual exigían también la implementación de políticas locales de gestión integral de residuos orientadas a la recuperación, reciclado y valorización de los desechos (Nuevo Ambiente, s. f.). Los principales actores interpelados por Nuevo Ambiente fueron Ceamse y el gobierno provincial. Al municipio de La Plata -el distrito del que formaban parte y que mayor cantidad de residuos enviaba al relleno- exigieron comenzar a implementar políticas de residuos.

La particularidad de Nuevo Ambiente es que, a diferencia del otro caso, utilizaron de forma combinada y sostenida en el tiempo estrategias de mediación, confrontación y expansión de la arena de decisión —como la judicialización y la presentación de propuestas en el poder legislativo local- para llevar sus demandas ante las autoridades. En este proceso, además, otorgaron mayor valor a las estrategias de expansión de la arena de decisión —como la judicialización—que en el caso de González Catán.

Yo creo que tuvo una organización desde lo judicial [...]. Entonces, al avanzar por ese camino se fueron condicionando mucho más las respuestas [...] y se fue como cercando un poco más a las estructuras que mantienen el statu quo, ¿viste? [...]. El cambio que se produjo en el área La Plata y Gran La Plata, tuvo un poco que ver con eso, tuvo 
que ver con las propias acciones judiciales que se fueron Ilevando (comunicación personal, Integrante de Organización Social, La Plata, 3 de junio 2012).

En 2004, Nuevo Ambiente, con una organización vecinal de la zona -Centro Vecinal de Punta Lara-, judicializó el reclamo presentando ante un juzgado provincial un recurso de amparo contra la Ceamse por violación a la prohibición del ingreso de residuos de distritos lejanos y la falta de estudios de impacto ambiental para la ampliación. Luego de una serie de apelaciones por parte de la empresa, la causa Ilegó a la Suprema Corte de Justicia de la Provincia, la mayor instancia judicial a la que se puede llegar en el ámbito provincial, que fue clave generando espacios de interacción entre las partes.

En este sentido, la judicialización, como estrategia de expansión de la arena de decisión, sirvió para presionar a la Ceamse, algo que reconocen las personas entrevistadas de otras organizaciones que también se sumaron al reclamo: "Con la justicia ya habiendo fallado en primera y segunda instancia $y$, luego, con el acuerdo de la Corte, se convino en que [el relleno] no podía crecer más. Se le puso como un corsé a las aspiraciones de Ceamse de

[ 82 ] continuar creciendo acá (comunicación personal, Integrante de Organización Social, La Plata, 3 de junio, 2012).

Estas acciones judiciales desarrolladas de forma sostenida en el tiempo permitieron a la organización presionar también al gobierno provincial, un actor al que se le exigió buscar una solución al problema de los residuos a partir de las intervenciones de la Suprema Corte de Justicia de la provincia:

Nosotros siempre fuimos contra CEAMSE, no contra la provincia... a la provincia la trae la Corte, no nosotros [...]. Cuando la Corte nos llama a todos, a nosotros para ver qué queríamos, a la Ceamse para ver cómo se podía hacer eso [...] si se podía o no [...] ahí, la Ceamse dice que también tiene que venir la provincia (comunicación personal, Integrante de Nuevo Ambiente, La Plata, 10 de marzo, 2018).

Así, la definición de la política de residuos y de la situación particular del relleno de Punta Lara dejó de estar exclusivamente en la esfera de la Ceamse y la provincia, ya que ahora un actor judicial también era parte de esto. A raíz de ello, en diciembre de 2006 se firmó un convenio entre la Ceamse y las organizaciones sociales que obligaba a la empresa a no recibir más residuos de municipios lejanos en el predio y a cerrarlo definitivamente, 
e intimaba al gobierno provincial a construir una planta de tratamiento de residuos para viabilizar dicho cierre (Nuevo Ambiente, s. f.).

Es interesante destacar que Nuevo Ambiente desplegó también la estrategia de confrontación desarrollando acciones contenciosas como la movilización, la protesta, el corte de calle y el bloqueo de la entrada al relleno sanitario (El Día, 2006, enero 4; Página 12, 2006, octubre 2; Nuevo Ambiente, s. f.). Estas acciones contenciosas eran complementarias a las acciones de mediación y expansión de la arena de decisión que fueron sostenidas con constancia a lo largo del tiempo por la organización.

Para forjar un cambio en la política en el ámbito local e instaurar políticas de gestión integral de residuos, Nuevo Ambiente también apeló a mediación y a la expansión de la arena de decisión para presionar al ejecutivo municipal, y lo hizo ampliando el juego hacia el legislativo local. En 2009 presentaron una propuesta al órgano legislativo local, la cual fue votada con mayoría y sancionada, dando origen a la Ordenanza 10661 de Basura Cero, la cual estableció la gestión integral de los desechos, haciendo obligatoria la recolección diferenciada de estos y la apertura de plantas de clasificación a cargo de cooperativas de recuperadores urbanos. Esas presentación, mediación y expansión se dieron casi en simultáneo, pues para ello las organizaciones se acercaron a un concejal que la presentó formalmente en el recinto: «La ordenanza de basura cero, prácticamente la escribimos nosotros, pero la presentó un concejal [...] no la presentamos nosotros» (comunicación personal, Integrante de Nuevo Ambiente, La Plata, marzo 10, 2018).

Una vez aprobada la ordenanza, quedaron establecidos nuevos parámetros para la gestión de los residuos que el ejecutivo debía incorporar y se implementaron programas afines a la nueva normativa - como el Programa Bolsa Verde- y se inauguraron centros de separación y clasificación de residuos (Nuevo Ambiente, s. f.). Nuevo Ambiente continuó con la estrategia de mediación articulando de forma constante con el gobierno municipal y marcando las eventuales deficiencias de implementación del programa (Nuevo Ambiente, 2015, octubre 30; 2015, noviembre 9).

No obstante, la historia del reclamo por los residuos no culminó con la sanción de la Ordenanza N. ${ }^{\circ} 10$ 661/2009, sino que la organización siguió presionando para que el convenio firmado por la Ceamse y la provincia 
en el ámbito judicial en 2006 se cumpliera. Con el paso de los años, el estancamiento de ese convenio provocó el rechazo de Nuevo Ambiente, el cual, además de seguir con el proceso judicial, decidió sumar un nuevo actor para que intercediera en el conflicto y contribuyera al desarrollo de la prometida planta de tratamiento que permitiera el cierre definitivo del relleno: el Defensor del Pueblo de la Provincia de Buenos Aires. Así, lo expresaba un entrevistado al referirse a esta nueva instancia de expansión del conflicto hacia otras arenas estatales: «Nosotros Ilamamos al Defensor para que interceda, para que sea una instancia de diálogo entre aquellos a los que convocaba la Corte por este tema [...]. Ahí recién se involucró el Defensor, pero porque nosotros lo llamamos» (comunicación personal, Integrante de Nuevo Ambiente, La Plata, 10 de marzo, 2018).

Dado que existía una sentencia judicial que obligaba a la provincia y a la Ceamse a construir la mencionada planta para poder cerrar el relleno, el Defensor propuso a todos los actores sociales y estatales involucrados evaluar posibles caminos para concretar la construcción de la misma. Allí, Ceamse propuso construir una planta de tratamiento para ese territorio que reduzca la cantidad de basura enterrada y permita cerrar el relleno. La planta fue construida y es operada por Ceamse. Funcionó a modo de prueba piloto desde diciembre de 2017 (El Día, 2017, diciembre 8; Infobae, 2017, diciembre 12) a marzo de 2019, cuando empezó a recibir el total de los residuos que iban al relleno de Punta Lara, consecuentemente cerrado al día de hoy (Infocielo, 2019, marzo 29).

\section{Conclusión}

Los casos analizados muestran que hay tres estrategias principales que pueden utilizar las organizaciones de base en procesos de incidencia: mediación, confrontación y expansión de las arenas de decisión. En los casos se observó que la combinación sostenida en el tiempo de estas estrategias favorece la incidencia, propiciando mejores y mayores efectos en las políticas públicas. Esto se destacó especialmente en el caso de Nuevo Ambiente, que con la combinación de las distintas estrategias logró generar una nueva política de residuos para su territorio: se construyó una planta de tratamiento de residuos que permitió cerrar el relleno sanitario de Punta Lara. En el caso de la Asamblea de Vecinos Autoconvocados, la estrategia sostenida en el tiempo fue principalmente la de confrontación en desmedro de la mediación y la expansión de la arena de decisión. El resultado obtenido en 
términos de incidencia fue la modificación parcial de la política de residuos por la reducción de la cantidad de desechos que ingresan al relleno, sin la proyección ni decisión estatal de su eventual clausura.

En estas líneas finales, se reconoce que las estrategias de acción son solo una dimensión factible de ser estudiada en la investigación de procesos de incidencia. No obstante, en algunos casos, las estrategias desplegadas por los actores pueden contribuir enormemente a comprender la incidencia, ya sea en estudios comparados o en estudios de casos. Asimismo, este tipo de investigación contribuye a una mejor comprensión de los actores que conforman ese gran agregado que solemos Ilamar «organizaciones sociales» y «políticas públicas». En relación con estas, es necesario tener siempre presente un resguardo especial para su estudio.

Tal como muestran los casos aquí analizados y la literatura, las organizaciones y las políticas públicas no se desarrollan en espacios sociales estancos u homogéneos, e interactúan constantemente con otras arenas sociales y estatales. Esto es fundamental para entender el despliegue de estrategias de mediación y expansión, y también para comprender la capacidad de incidencia de este tipo de organizaciones más allá de la sola confrontación en otros casos similares de incidencia en políticas públicas del resto de Argentina y otros países de la región.

\section{Referencias bibliográficas}

1. Alarcón, Cristian. (2006, julio 22). Un piquete en La Matanza por el agua que contaminan los residuos. Página 12. Recuperado de https://www.pagina12.com.ar/ imprimir/diario/sociedad/3-70324-2006-07-22.html

2. Andretta, Massimiliano; Piazza, Gianni \& Subirats, Anna. (2015). Urban Dynamics and Social Movements. In: Della Porta, Donatella \& Diani, Mario (eds.). The Oxford Handbook of Social Movements (pp. 200-218). Oxford: Oxford University.

3. ANRed, (2006, enero 12). Vecinos de González Catán denuncian a la Ceamse. Recuperado de http://www.anred.org/?p=2637

4. Aranda, Darío. (2004, enero 31). Un basural que deja una herencia. Página 12. Recuperado de http://www.pagina12.com.ar/diario/sociedad/3-30975-2004-01-31. html

5. Argentina. La Matanza. Honorable Concejo Deliberante. Expediente 1000. (2 de noviembre de 2007).

6. Argentina. Municipalidad de La Plata. Concejo Deliberante. Ordenanza 10661. (16 de diciembre de 2009). Recuperado de http://www.concejodeliberante. laplata.gov.ar/digesto/or11000/or10661.asp?ver $=$ \& resol = 
7. Argentina. Provincia de Buenos Aires. Juzgado Federal en lo Criminal y Correccional N. ${ }^{\circ} 3$ de Morón. Causa 2993. (16 de junio de 2006).

8. Asamblea de Vecinos Autoconvocados contra la CEAMSE de González Catán (2007). Programa Catán sin Basura. [Folleto].

9. Boucher, Anna. (2013). Bureaucratic Control and Policy Change: A Comparative Venue Shopping Approach to Skilled Immigration Policies in Australia and Canada. Journal of Comparative Policy Analysis: Research and Practice, 15 (4), pp. 349-367. DOI: 10.1080/13876988.2012.749099

10. Bryant, Raymond L. \& Bailey, Sinéad. (1997). Third World Political Ecology. London: Routledge.

11. Bullard, Robert D. \& Johnson, Glenn S. (2000). Environmental Justice: Grassroots Activism and Its Impact on Public Policy Decision Making. Journal of Social Issues, 56 (3), pp. 555-578. DOI: 10.1111/0022-4537.00184

12. Burstein, Paul \& Linton, April. (2002). The Impact of Political Parties, Interest Groups, and Social Movement Organizations on Public Policy: Some Recent Evidence and Theoretical Concerns. Social Forces, 81 (2), pp. 381-408. DOI: 10.1353/ sof.2003.0004

13. Burstein, Paul; Einwohner, Rachel L. \& Hollander, Jocelyn A. (1995). The Success of Political Movements: A Bargaining Perspective. En: Jenkins, J. Craig \& Klandermans, Bert (eds.). The Politicis of Social Protest: Comparative Perspectives on States and Social Movements (pp. 135-144). London: UCL.

[ 86 ] 14. Capano, Giliberto \& Howlett, Michael. (2009). Introduction: The Determinants of Policy Change: Advancing the Debate. Journal of Comparative Policy Analysis, 11 (1). DOI: 10.1080/13876980802648227

15. Cortez, Maurizio y Maillet, Antoine. (2018). Trayectoria multinivel de una coalición promotora e incidencia en la agenda política nacional. El caso del conflicto de Pascua Lama y la ley de glaciares en Chile. Colombia Internacional, 94, pp. 3-25. DOI: 10.7440/colombiaint94.2018.01

16. Christel, Lucas G. \& Gutiérrez, Ricardo A. (2017). Making Rights Come Alive: Environmental Rights and Modes of Participation in Argentina. Journal of Environment and Development, 26 (3), pp. 322-347. DOI: 10.1177/1070496517701248

17. Delamata, Gabriela. (2016). Una década de activismo judicial en las villas de Buenos Aires. Revista Direito e Práxis, 7 (14), pp. 567-587. DOI: 10.12957/ dep.2016.22952

18. Diani, Mario. (2003). "Leaders» Or Brokers? Positions and Influence in Social Movement Networks. In: Diani, Mario \& McAdam, Doug (eds.). Social Movements and Networks: Relational Approaches to Collective Action. DOI: 10.1093/0199251789.003.0005

19. El Día. (2003, diciembre 6). Ahora Ilega basura de Berazategui y Varela. Recuperado de http://www.eldia.com/nota/2003-12-6-ahora-llega-basura-deberazategui-y-varela 
Modelando las políticas públicas. Estrategias de las organizaciones de base...

20. El Día. (2006, enero 4). Vecinos cortaron acceso al Ceamse por la «contaminación ambiental». Recuperado de http://www.eldia.com/nota/2006-1-4vecinos-cortaron-acceso-al-ceamse-por-la-contaminacion-ambiental

21. El Día. (2007, septiembre 12). Movilización por el cierre de Ceamse en La Matanza. Recuperado de http://www.eldia.com/nota/2007-9-12-movilizacion-por-elcierre-de-ceamse-en-la-matanza

22. El Día. (2017, diciembre 8). Empezó a funcionar la nueva planta de residuos de CEAMSE, que estará a pleno en marzo. Recuperado de https:/www.eldia.com/ nota/2017-12-8-2-27-18-empezo-a-funcionar-la-nueva-planta-de-residuos-de-ceamseque-estara-a-pleno-en-marzo-la-ciudad

23. El1Digital. (2010, septiembre 20). Los concejales convalidaron el CARE. Recuperado de http://www.el1digital.com.ar/articulo/view/17972/

24. Evans, Peter; Rueschemeyer, Dietrich \& Skocpol, Theda (eds.). (1985). Bringing the State Back In. Cambridge: Cambridge University. DOI: 10.1017/ CBO9780511628283

25. Gutiérrez, Ricardo A. e Isuani, Fernando. (2014). La emergencia del ambientalismo estatal y social en Argentina. Revista de Administração Pública, 48 (2), pp. 295-332. DOI: 10.1590/0034-76121700

26. Infobae. (2017, diciembre 12). Presentaron una nueva planta para tratamiento de residuos en Ensenada. Recuperado de http://www.infobae.com/ sociedad/2017/12/12/presentaron-una-nueva-planta-para-tratamiento-de-residuos-enensenada/

27. Infocielo (2019, marzo 29). Histórico: la CEAMSE cerró el relleno de Punta Lara y con la basura generarán electricidad. Recuperado de https://infocielo.com/ nota/102998/historico-la-ceamse-cerro-el-relleno-de-punta-lara-y-con-la-basurageneraran-electricidad/

28. Jenkins-Smith, Hank C. \& Sabatier, Paul A. (1994). Evaluating the Advocacy Coalition Framework. Journal of Public Policy, 14 (2), pp. 175-203. DOI: 10.1017/ S0143814X00007431

29. Kingdon, John. (1995). Agendas, Alternatives and Public Policy. New York: Harper Collins.

30. König, Thomas; Tsebelis, George \& Debus, Marc (eds.). (2011). Reform Processes and Policy Change: Veto Players and Decision-Making in Modern Democracies. Virginia: Springer. DOI: 10.1007/978-1-4419-5809-9

31. Kotler, Philip \& Zaltman, Gerald. (1971). Social Marketing: An Approach to Planned Social Change. Journal of Marketing, 35 (3), pp. 3-12. DOI: 10.1177/002224297103500302

32. La Nación. (2006, noviembre 3). Tras los incidentes, exigen el cierre del Ceamse. Recuperado de https://www.lanacion.com.ar/855415-tras-los-incidentesexigen-el-cierre-del-ceamse 
33. Leech, Beth L. (2010). Lobbying and Influence. In: Maisel, L. Sandy; Berry, Jeffrey \& Edwards, George III (eds.). Oxford Handbook of American Political Parties and Interest Groups (pp. 534-551). Oxford: Oxford University.

34. Lindblom, C. E. (1992). La ciencia de «salir del paso». En: Aguilar Villanueva, Luis (ed.). La hechura de las políticas (pp. 201-225). México, D. F.: Porrúa.

35. Lowi, Theodore. (1996). Políticas Públicas, estudios de caso y teoría política. En: Aguilar Villanueva, Luis (ed.). La hechura de las políticas (pp. 89-117). México, D. F.: Porrúa.

36. Mahoney, James. (2001). Path-Dependent Explanations of Regime Change: Central America in Comparative Perspective. Studies in Comparative International Development, 36 (1), pp. 111-141. DOI: 10.1007/BF02687587

37. Mann, Michael. (2008). Infrastructural Power Revisited. Studies in Comparative International Development, 43 (3-4), pp. 355-365. DOI: 10.1007/ s12116-008-9027-7

38. McAdam, Doug \& Su, Yang. (2002). The War at Home: Antiwar Protests and Congressional Voting, 1965 to 1973. American Sociological Review, 67 (5), pp. 696721. DOI: $10.2307 / 3088914$

39. McCarthy, John D. \& Wolfson, Mark. (1996). Resource Mobilization by Local Social Movement Organizations: Agency, Strategy, and Organization in the Movement Against Drinking and Driving. American Sociological Review, 61 (6), pp. 1070-1088. DOI: 10.2307/2096309

[ 88 ] 40. Merlinsky, María Gabriela. (2013). Política, derechos y justicia ambiental: el conflicto del Riachuelo. Buenos Aires: Fondo de Cultura Económica.

41. Mitlin, Diana. (2008). With and Beyond the State Co-Production as a Route to Political Influence, PowerandTransformationforGrassrootsOrganizations. Environment and Urbanization, 20 (2), pp. 339-360. DOI: 10.1177/0956247808096117

42. Molina, Anabella. (2017, 26-28 de julio). Coaliciones: un veto para la paridad de género en los Gabinetes de América Latina. 9. ${ }^{\circ}$ Congreso Latinoamericano de Ciencia Política. Asociación Latinoamericana de Ciencia Política (Alacip), Montevideo.

43. Moore, Sarah A. (2009). The Excess of Modernity: Garbage Politics in Oaxaca, Mexico. The Professional Geographer, 61 (4), pp. 426-437. DOI: 10.1080/00330120903143375

44. Moreno Mena, José Asención y Arballo Meza, Rosa Amelia. (2003). Experiencias en Políticas Públicas de las ONGs en Baja California. Estudios Fronterizos, 4 (8), pp. 73-103.

45. Najam, Adil. (2000). The Four-C's of Third Sector: Government Relations. Cooperation, Confrontation, Complementarity, and Co-Optation. Nonprofit Management \& Leadership, 10 (4), pp. 375-396. DOI: 10.1002/nml.10403

46. Nuevo Ambiente (s. f.). No + CEAMSE en Punta Lara. Un poco de historia sobre la movilización popular por una Gestión Integral de los Residuos Sólidos Urbanos. Recuperado de http://www.nuevoambiente.org/no-mas-ceamse/ 
47. Nuevo Ambiente. (2015, noviembre 9). Residuos en La Plata, reiteramos solicitud de garantías. Recuperado de http://www.nuevoambiente.org/2015/11/09/ residuos-en-la-plata-reiteramos-solicitud-de-garantias/

48. Nuevo Ambiente. (2015, octubre 30). Se solicitó garantías para recolección Bolsas Verdes y se abrieron sobres de la licitación. Recuperado de http://www. nuevoambiente.org/2015/10/30/se-solicito-garantias-para-recoleccion-bolsas-verdesy-se-abrieron-sobres-de-la-licitacion/

49. Observatorio del Conurbano Bonaerense. (s. f). Región Metropolitana de Buenos Aires. Recuperado de http://observatorioconurbano.ungs.edu.ar/?page_ $\mathrm{id}=2708$

50. Offe, Claus \& Wiesenthal, Helmut. (1980). Two Logics of Collective Action: Theoretical Notes on Social Class and Organizational Form. Political Power and Social Theory, 1 (1), pp. 67-115.

51. Página 12. (2006, octubre 2). «CEAMSE contamina el suelo, el aire, el agua y la moral». Recuperado de http://www.pagina12.com.ar/diario/ ultimas/20-73902-2006-10-02.html

52. Pierson, Paul \& Skocpol, Theda. (2008). El institucionalismo histórico en la Ciencia Política Contemporánea. Revista Uruguaya de Ciencia Política, 17 (1), pp. 7-38.

53. Pierson, Paul. (1993). When Effect Becomes Cause: Policy Feedback and Political Change. World Politics, 45 (4), pp. 595-628. DOI: 10.2307/2950710

54. Pralle, Sarah B. (2003). Venue shopping, political strategy, and policy change: The internationalization of Canadian Forest Advocacy. Journal of Public Policy, 23 (3), pp. 233-260. DOI: 10.1017/S0143814X03003118

55. Pralle, Sarah B. (2006). Branching Out, Digging In: Environmental Advocacy and Agenda Setting. Washington, D. C.: Georgetown University.

56. Real-Dato, José. (2009). Mechanisms of Policy Change: A Proposal for a Synthetic Explanatory Framework. Journal of Comparative Policy Analysis: Research and Practice, 11 (1), pp. 117-143. DOI: 10.1080/13876980802648268

57. Ryan, Daniel Eduardo. (2011). Democratic Governance and the Courts: The Political Sources of the Judicialization of Public Policy in Argentina. (PhD. Dissertation). University of Texas.

58. Sabatini, Francisco. (1997). Conflictos ambientales y desarrollo sostenible de las regiones urbanas. Revista EURE, xxII (68), pp. 77-91.

59. Semanario Quinto Poder. (2015, marzo 2). «El CARE fue la gran mentira de La Matanza, el gran negociado». Recuperado de http://semanarioquintopoder. $\mathrm{com} / \mathrm{p}=5214$

60. Sorroche, Santiago. (2017). Experiencias replicables. Análisis de las vinculaciones entre cooperativas de cartoneros, agencias estatales y ONG en el Gran Buenos Aires. Revista de Estudios Sociales, 61, pp. 58-68. DOI: 10.7440/ res61.2017.05 
61. Steedly, Homer R. \& Foley, John W. (1979). The Success of Protest Groups: Multivariate Analyses. Social Science Research, 8 (1), pp. 1-15. DOI: 10.1016/0049089X(79)90011-5

62. Tarrow, Sidney \& Tilly, Charles. (2009). Contentious Politics and Social Movements. In: Boix, Carles \& Stokes, Susan (eds.). The Oxford Handbook of Comparative Politics. Oxford: Oxford University. DOI: 10.1093/ oxfordhb/9780199566020.003.0019

63. Tarrow, Sidney. (2011). Power in Movement: Social Movements, Collective Action and Politics. Cambridge: Cambridge University. DOI: 10.1017/ CBO9780511973529

64. Tilly, Charles. (2001). Mechanisms in Political Processes. Annual Review of Political Science, 4, pp. 21-41. DOI: 10.1146/annurev.polisci.4.1.21

65. True, James; Jones, Bryan y Baumgartner, Frank. (2010). Teoría del equilibrio interrumpido. En: Sabatier, Paul A. (ed.). Teorías del proceso de las políticas públicas (pp. 167-202). Buenos Aires: Proyecto de Modernización del Estado.

66. Uphoff, Norman. (1993). Grassroots Organizations and NGOs in Rural Development: Opportunities with Diminishing States and Expanding Markets. World Development, 21 (4), pp. 607-622. DOI: 10.1016/0305-750X(93)90113-N

67. Vedung, Evert. (1998). Policy Instruments: Typologies and Theories. In: Bernelmans-Videc, Marie-Louise; Rist, Ray \& Vedung, Evert (eds.). Carrots, Sticks and Sermons. Policy Instruments and their Evaluation (pp. 21-58). New Brunswick and [ 90 ] London: Transaction. DOI: 10.4324/9781315081748-2

68. Walsh, Ed J.; Warland, Rex \& Smith, D. Clayton. (1997). Don't Burn It Here: Grassroots Challenges to Trash Incinerators. Pennsylvania: Pennsylvania State University.

69. Ward, Matthew. (2017). Opportunity, Resources, and Threat: Explaining Local Nativist Organizing in the United States. Sociological Perspectives, 60 (3), pp. 459-478. DOI: 10.1177/0731121416655994

70. Weible, Christopher M.; Sabatier, Paul A.; Jenkins $\square$ Smith, Hank C.; Nohrstedt, Daniel; Henry, Adam Douglas \& deLeon, Peter. (2011). A Quarter Century of the Advocacy Coalition Framework: An Introduction to the Special Issue. Policy Studies Journal, 39 (3), pp. 349-360. DOI: 10.1111/j.1541-0072.2011.00412.x

71. Whiteley, Paul F. \& Winyard, Stephen L. (1987). Pressure for the Poor: The Poverty Lobby and Policy Making. London: Methuen.

72. Ydersbond, Inga Margrete. (2014). Multilevel «Venue Shopping»: The Case of EU's Renewables Directive. Interest Groups and Advocacy, 3 (1), pp. 30-58. DOI: 10.1057/iga.2013.12

73. Young, Sokphea. (2016). Movement of Indigenous Communities Targeting an Agro-Industrial Investment in North-Eastern Cambodia. Asian Journal of Social Science, 44 (1-2), pp. 187-213. DOI: 10.1163/15685314-04401008 